\title{
Consumo de Pornografia e Relacionamento Amoroso: uma Revisão Sistemática do Período 2006-2015
}

\section{Pornography Consumption and Romantic Relationships: a Systematic Review (2006-2015)}

\section{Cynthia Perovano Camargo Baumel1, Valeschka Martins Guerra², Agnaldo Garcia $^{3}$, Alini Gusmão Rosário ${ }^{4}$}

\begin{abstract}
Resumo
Trata-se de revisão sistemática para investigar as definições de pornografia utilizadas por pesquisadores e os efeitos do consumo desse material nos relacionamentos amorosos. Buscaram-se artigos teóricos ou empíricos, nos idiomas espanhol, inglês e português, publicados em periódicos nacionais e internacionais, indexados nas plataformas eletrônicas Doaj, SciELO e Scopus, que contivessem, em qualquer parte do texto, os termos "pornografia" e "relacionamento". Retornaram 433 documentos que, aplicados os critérios de inclusão e exclusão, resultaram em 45 artigos. Na amostra estudada, material sexualmente explícito parece ser a definição mais aceita de pornografia. Em relação aos aspectos negativos percebidos do consumo de pornografia ao relacionamento, foram elencadas as categorias: prejuízos à saúde, prejuízos ao relacionamento, violência contra as mulheres e aspectos socioculturais. Em relação aos aspectos positivos, foram levantadas as categorias: sexualidade, desenvolvimento pessoal e benefícios ao relacionamento. Os possíveis impactos do consumo de pornografia nos relacionamentos são ainda bastante imprecisos e ambíguos.
\end{abstract}

Palavras-chave: Pornografia. Relacionamento. Casal. Sexualidade. Revisão.

\begin{abstract}
This systematic review aims to investigate pornography definitions used by researchers and the effects of the consumption of this material in romantic relationships. We searched for theoretical or empirical papers published in Brazilian and international journals indexed in the DOAJ, SciELO and Scopus electronic platforms, which contained, in any part of the text, the terms "pornography" and "relationship" in Spanish, English or Portuguese. This search resulted in 433 documents that, after application of the inclusion and exclusion criteria, resulted in 45 papers. Within the sample, sexually explicit material seems to be the most accepted definition of pornography. Regarding the perception of negative aspects of pornography consumption on the relationship, the following categories were listed: health damage, relationship impairment, violence against women and social and cultural aspects. In relation to the
\end{abstract}

\footnotetext{
${ }^{1}$ Universidade Federal do Espírito Santo, Espírito Santo, Brasil. E-mail: cynthiaperovano@ufes.br.

${ }^{2}$ Universidade Federal do Espírito Santo, Espírito Santo, Brasil. E-mail: valeschkamartins@gmail.com.

${ }^{3}$ Universidade Federal do Espírito Santo, Espírito Santo, Brasil. E-mail: agnaldo.garcia@uol.com.br.

${ }^{4}$ Universidade Federal do Espírito Santo, Espírito Santo, Brasil. E-mail: alini.gr@gmail.com.
} 
positive aspects, the categories surveyed were: sexuality, personal development and benefits to the relationship. The possible impacts of pornography consumption in relationships are still quite vague and ambiguous.

Keywords: Pornography. Relationship. Couple. Sexuality. Review. 


\section{Introdução}

O consumo de pornografia em nossa sociedade é inegável, podendo ser facilmente encontrada em revistas, fotografias, vídeos e tantos outros meios. Com o auxílio de novas tecnologias, como internet, smartphones e tablets, o acesso ao pornô está a um clique de distância (Grov, Gillespie, Royce \& Lever, 2011; Popovic, 2011). Entre os 50 sites mais populares de todo o mundo, quatro são de conteúdo pornográfico. O Pornhub, site que oferece conteúdo gratuito, aparece em $11^{\circ}$ lugar, contabilizando 33,5 bilhões de visitas no último ano, ultrapassando eBay, MSN e Netflix em números de acesso (SimilarWeb, 2018). Segundo informações divulgadas pelo site, $71,6 \%$ desse acesso ocorre por smartphones e o Brasil é o $12^{\circ}$ colocado no mundo em números de visitantes por dia (Pornhub Team, 2018). Nesse sentido, este artigo de revisão sistemática tem como objetivo investigar as definições de pornografia utilizadas por pesquisadores e os efeitos percebidos do consumo desse material nos relacionamentos amorosos.

A palavra pornografia vem do grego pornographos e foi originalmente usada pela primeira vez para descrever a vida, costumes, hábitos e maneiras das prostitutas e seus clientes (Popovic, 2011). O dicionário Michaelis utiliza termos como devassidão, imoralidade e libertinagem para definir pornografia, como "qualquer coisa que vise explorar o sexo de maneira vulgar e obscena" (Michaelis, 2015). Palavras como pornô, material erótico, filme com conteúdo explícito, produção para maiores e conteúdo adulto, entre outras, também são utilizadas como sinônimos para pornografia. Diante dessa diversidade de possibilidades, faz-se necessário investigar as definições de pornografia utilizadas por pesquisadores no meio acadêmico.

Abordar as representações de comportamentos sexuais por meios diversos, como palavras e imagens, é também falar sobre a sexualidade humana. A maneira como cada indivíduo a experiencia é única, construída no contexto social, cultural, histórico e econômico no qual está imerso. Sentimentos, crenças, valores e expectativas determinam a forma como homens e mulheres amam e praticam sexo (Lins, 2012). A sexualidade permeia a vida, e a forma como é construída impactará nas atitudes de uma pessoa com relação à sexualidade e a sua percepção desta na sociedade, por exemplo, para avaliar uma manifestação sexual como pornográfica e a percepção dos impactos de seu consumo nos relacionamentos.

Andrade, Garcia e Cano (2009) afirmam que se relacionar romanticamente é parte natural do processo de vida da maioria das pessoas e elemento comum em diferentes culturas, sociedades e momentos históricos. Somos constituídos pelos relacionamentos que estabelecemos e por isso é tão importante investigar como se dão esses vínculos, o que os afeta positiva e negativamente. Esses autores apontam, ainda, que a satisfação com o relacionamento é um importante preditor da avaliação do bem-estar psicológico do indivíduo. Pessoas satisfeitas no relacionamento amoroso, em geral, vivenciam de maneira positiva os demais contextos da vida. Aspectos específicos como sexo, intimidade, comprometimento e comunicação parecem contribuir com a avaliação 
global da qualidade dos relacionamentos amorosos. Seria o consumo de pornografia mais um componente cuja influência nos relacionamentos é possível avaliar?

Diante do exposto, nota-se a relevância social de um estudo dessa natureza. Se por um lado temos um enorme mercado de produtos pornográficos e similares em franca expansão (Abeme, 2014), por outro as consequências dessa oferta precisam ser investigadas. A disponibilidade desse conteúdo, especialmente na internet, permite o acesso com mais facilidade e em grande diversidade. $\mathrm{O}$ aumento do número de materiais publicados parece indicar um interesse científico crescente na interface entre pornografia e relacionamentos. Duas abordagens contrárias surgem e parecem competir entre si - de que a pornografia teria efeitos bons ou ruins (Popovic, 2011; Staley \& Prause, 2013).

Algumas pesquisas vêm apontando efeitos negativos do consumo de pornografia, como a associação entre uso de pornografia e atitudes de apoio à violência contra a mulher, incluindo um aumento da objetificação, mais aceitação do estupro e um aumento de atitudes agressivas e de agressão (Bonomi et al., 2014; Braithwaite, Aaron, Dowdle, Spjut \& Fincham, 2015; DeKeseredy, 2015; Hald \& Malamuth, 2015). Outras apontam como consequência, ainda, a redução da satisfação dos sujeitos com seu relacionamento (Pyle \& Bridges, 2012; Muusses, Kerkhof \& Finkenauer, 2015; Szymanski, Feltman \& Dunn, 2015; Weinstein, Zolek, Babkin, Cohen \& Lejoyeux, 2015), o aumento dos comportamentos de risco (Crimmins \& Seigfried-Spellar, 2014; Wu et al., 2014; Braithwaite et al., 2015; Silvera, Grov, Stein, Hagerty \& Marmor, 2015) e do uso excessivo, associados à dependência (Stewart \& Szymanski, 2012; Hilton, 2013; Olmstead, Negash, Pasley \& Fincham, 2013; Şenormanci, Konkan, Güçlü \& Şenormanci, 2014).

Em contrapartida, algumas pesquisas concluíram que a pornografia pode ser uma ferramenta útil de aprendizado e de expansão do repertório sexual dos indivíduos (Chi, Yu \& Winter, 2012; Mowlabocus, Harbottle \& Witzel, 2013; Olmstead et al., 2013; Elder, Morrow \& Brooks, 2015) e auxilia a aumentar a excitação e satisfação sexual em certas circunstâncias (Grov et al., 2011; Maddox, Rhoades \& Markman, 2011; Muusses et al., 2015), podendo ser uma forma saudável de realização de fantasias sexuais (Fahs \& Gonzalez, 2014), além de melhorar a comunicação e aproximar os parceiros (Benjamin \& Tlusten, 2010; Grov et al., 2011; Popovic, 2011; Elder et al., 2015), revelando-se como um recurso potente para uma vida sexual mais prazerosa e bem informada (Guerra, Andrade \& Dias, 2004).

A presente pesquisa pretende reunir os resultados de múltiplos estudos, descrevendo a produção científica sobre o assunto: áreas do conhecimento relacionadas, características das pesquisas (população, amostra, etc.), definição de pornografia e efeitos percebidos. Pretende, assim, colaborar na ampliação dessa área de conhecimento, sistematizando os artigos referentes a esse assunto, fornecendo elementos para uma maior compreensão e melhor intervenção na maneira como o consumo de pornografia tem tido influência nos relacionamentos amorosos. Nesse sentido, a revisão sistemática é um método que permite maximizar o potencial de uma busca, reunindo de forma organizada os resultados de pesquisas e permitindo analisar criticamente as informações 
relevantes encontradas (Costa \& Zoltowski, 2014).

\section{Método}

Foram seguidas as oito etapas básicas sugeridas por Costa e Zoltowski (2014) para a construção desta revisão sistemática. $\mathrm{O}$ percurso metodológico será apresentado a seguir, segundo essas etapas.

\section{Delimitação da questão a ser pesquisada}

Em consonância com o objetivo da pesquisa, a questão norteadora da revisão foi "que efeitos são percebidos nos relacionamentos amorosos do consumo de pornografia?" Também se procurou identificar e descrever como a pornografia tem sido definida por pesquisadores.

\section{Escolha das fontes de dados}

Uma primeira busca foi realizada no Portal de Periódicos da Capes, uma biblioteca virtual que reúne e disponibiliza diversas produções científicas nacionais e internacionais, que conta com diversas bases de dados eletrônicas em seu acervo. Como o Portal tem diversas bases indexadas, poderia fornecer um panorama de como esse tema vem sendo investigado.

Ao pesquisar no Portal, a base de dados que retornou um maior número de resultados foi a Scopus. Apesar dessa base se tratar da maior fonte referencial de literatura técnica e científica revisada por pares, muitos de seus periódicos têm acesso restrito.
Duas outras bases foram selecionadas, Doaj (Directory of Open Access Journals) e SciELO (Scientific Eletronic Library Online), por ambas disponibilizarem textos completos e de acesso gratuito. A busca foi refeita para proceder à seleção do material, utilizando-se as bases de dados específicas escolhidas: Scopus, Doaj e SciELO.

\section{Eleição das palavras-chave para a busca}

Os descritores buscaram abranger as duas direções desse estudo - pornografia e relacionamento. $\mathrm{O}$ termo pornografia foi utilizado sem adaptações, para que se pudesse recuperar as definições utilizadas. Para pesquisar relacionamento amoroso, diversas combinações de palavras seriam possíveis, como relacionamento íntimo e relacionamento romântico, por exemplo. Para simplificar a estratégia de busca, foi utilizado apenas o termo relacionamento, acrescentando a palavra casal. Referências à pornografia infantil, que não são foco desta revisão, foram excluídas.

A estratégia de busca utilizada foi: pornografia/pornography/pornografía AND (relacionamento/relationship/relacionamiento OR casal/couple/pareja) AND NOT criança*/ child $^{*} /$ niño* ${ }^{*}$ pesquisada em cada idioma separadamente.

\section{Busca e armazenamento dos resultados}

Foi realizada uma busca avançada nas bases selecionadas em janeiro de 2016 com tais termos e booleanos. Foram procurados artigos, publicados nos últimos 10 anos (2006-2015), nos idiomas português, inglês e espanhol que 
contivessem os termos buscados em qualquer lugar do texto. Todos os documentos encontrados foram salvos. Essa busca foi feita separadamente por dois juízes independentes que, ao confrontarem os achados, encontraram os mesmos resultados.

\section{Seleção de artigos pelo resumo, de acordo} com critérios de inclusão e exclusão

A partir da busca realizada, uma lista de referência dos artigos foi organizada (Scopus $\mathrm{N}=$ 164; Doaj N = 160; SciELO N = 109; Total = 433). Essa relação foi avaliada e 124 artigos foram excluídos, por estarem repetidos ou pelo ano de publicação extrapolar o intervalo considerado. $\mathrm{Na}$ Figura 1. Resumo da busca.

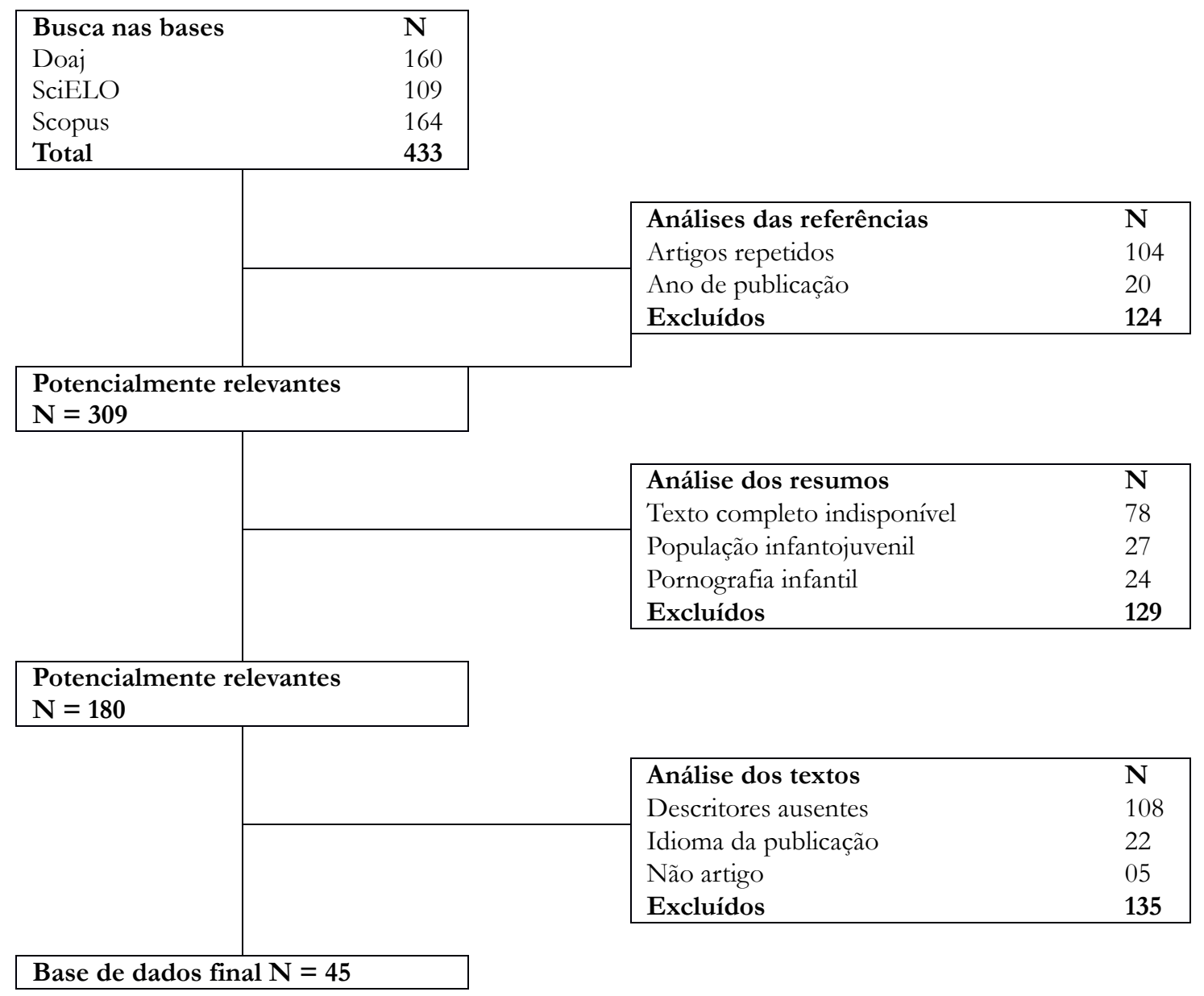

análise dos resumos, foram excluídos os que não disponibilizavam texto completo, investigavam população infantojuvenil ou tratavam de pornografia infantil, restando 180 documentos potencialmente relevantes. Esses documentos foram analisados segundo os critérios de exclusão: idioma diferente dos elencados, produção diferente de artigo científico e ausência dos descritores da busca. O termo "relacionamento" foi considerado apenas quando em um contexto de relação amorosa e/o sexual, excluindo as referências às relações entre coisas e ao estado civil. A base de dados final para a revisão foi de 45 artigos. O processo de busca pode ser resumido como mostra a Figura 1. 


\section{Extração dos dados dos artigos selecionados}

Os 45 artigos selecionados na revisão sistemática foram organizados em um software de planilha eletrônica, de forma a catalogar os dados de acordo com: ano de publicação, país de publicação, área de conhecimento, tipo de estudo, tamanho da amostra, métodos de coleta de dados, população, objetivos, definições de pornografia e relações negativas e positivas do consumo de pornografia com o relacionamento, entre outros.

As etapas 7 (avaliação dos artigos) e 8 (sintese e interpretação dos dados) serão apresentadas a seguir.

\section{Resultads e Discussão}

$\mathrm{Na}$ distribuição dos artigos por ano, pode-se observar um baixo número de produções no período de 2006-2009 (1,5/ano) e um salto no número de publicações no ano $2010 \quad$ (8 publicações), seguido por uma leve redução entre 2011-2014 (4,75/ano) e novo salto em 2015 (12 publicações). Essa ampliação do número de produções ao longo do período pode sugerir um aumento do interesse na temática por parte dos pesquisadores.

A maior parte das publicações está em língua inglesa (42 artigos; 93\%); seguida da língua portuguesa ( 2 artigos; 4\%) e da língua espanhola (1 artigo; $2 \%)$. Nota-se, na amostra estudada, a importância da língua inglesa na divulgação do conhecimento científico.

Foram encontrados artigos de várias partes do mundo, com destaque para os Estados Unidos, com 26 artigos (58\% do total); em seguida, temos Brasil, Canadá, China, Israel e Reino Unido, com dois artigos cada (4\%); e os demais países, com um artigo cada (2\%). Apesar do alto consumo de pornografia no Brasil, apenas um dos estudos encontrados focava a população brasileira (D'Abreu, 2013), de acordo com os critérios de inclusão e exclusão utilizados para a seleção dos artigos nessas bases de dados.

As publicações foram desenvolvidas por pesquisadores das mais variadas áreas de conhecimento. Para fins de sistematização, os artigos foram organizados segundo a classificação do Conselho Nacional de Pesquisa (CNPq) das áreas do conhecimento, tomando por base o autor principal de cada artigo. Assim, temos Ciências Sociais Aplicadas $(\mathrm{N}=10 ; 22 \%$ ) congregando publicações em Comunicação Social, Direito, Economia e Serviço Social; Ciências da Saúde $(\mathrm{N}=8 ; 18 \%)$, com Medicina e Saúde Coletiva; Ciências Humanas ( $\mathrm{N}=7 ; 16 \%$ ), com Educação, Filosofia e Sociologia; e Ciências Exatas e da Terra $(\mathrm{N}=1 ; 2 \%)$ com Estatística. Psicologia deveria ter sido incluída nas Ciências Humanas, mas optou-se por calcular essa área à parte, de modo a explicitar sua representatividade. Foram 19 artigos, $42 \%$ do total.

Em termos de áreas de estudo, a produção é bastante diversificada, com artigos versando sobre aspectos jurídicos, psicológicos, culturais, sociais, morais e de saúde. A área de conhecimento que concentrou o maior número de trabalhos publicados foi a Psicologia. Tal concentração aponta uma preocupação desse campo do saber nos impactos do consumo de pornografia nos relacionamentos.

No que se refere ao tipo de estudo realizado, destacam-se nessas publicações os relatos de pesquisas, com 37 artigos, que corresponde a $82 \%$ do total. Por outro lado, notase a escassez de investigações como a revisão de literatura $(\mathrm{N}=7 ; 16 \%)$ e a meta-análise $(\mathrm{N}=1$; 
2\%). Tal constatação reitera a necessidade de levantamentos como o presente estudo.

Com relação à população estudada, os universitários foram a maioria $(\mathrm{N}=15 ; 41 \%)$. Essa categoria é composta por artigos somente com mulheres, somente com homens e com ambos, estando em um relacionamento ou não. Talvez essa predominância seja em virtude de as pesquisas serem divulgadas nas universidades e, muitas vezes, oferecerem créditos em disciplinas aos estudantes. $\mathrm{O}$ Quadro 1 identifica a população e o tamanho da amostra de cada artigo. 
Quadro 1. População, tamanho de amostra, definição de pornografia e categorias por artigo

\begin{tabular}{|c|c|c|c|c|c|}
\hline \multirow[b]{2}{*}{ Artigo } & \multirow[b]{2}{*}{ População } & \multirow[b]{2}{*}{$\underset{\mathrm{ra}}{\mathrm{Amost}}$} & \multirow[b]{2}{*}{$\begin{array}{l}\text { Definição de } \\
\text { pornografia }\end{array}$} & \multicolumn{2}{|c|}{ Categorias } \\
\hline & & & & $\begin{array}{c}\text { Negativo } \\
\text { ao } \\
\text { relacionam } \\
\text { ento } \\
\end{array}$ & $\begin{array}{l}\text { Positivo ao } \\
\text { relacionam } \\
\text { ento }\end{array}$ \\
\hline $\begin{array}{l}\text { Benjamin \& } \quad \& \quad \text { Tlusten } \\
(2010)\end{array}$ & $\begin{array}{l}\text { mulheres em um } \\
\text { relacionamento }\end{array}$ & 20 & $\begin{array}{l}\text { material sexualmente } \\
\text { explícito, sem } \\
\text { intimidade }\end{array}$ & Re-, Vi & Ds, Re+ \\
\hline Bonomi et al. (2014) & $\begin{array}{l}\text { universitárias } \\
\text { (SF) }\end{array}$ & 655 & material hard-core & $\mathrm{Vi}, \mathrm{Sc}$ & - \\
\hline Bouffard (2010) & $\begin{array}{l}\text { universitários } \\
\qquad(\mathrm{SM})\end{array}$ & 325 & $\begin{array}{l}\text { material sexualmente } \\
\text { explícito }\end{array}$ & Vi & - \\
\hline Braithwaite et al. (2015) & $\begin{array}{l}\text { universitários } \\
\text { (SF/SM) }\end{array}$ & $\begin{array}{c}850 / \\
992\end{array}$ & $\begin{array}{l}\text { sem definição, com } \\
\text { exemplos }\end{array}$ & $\mathrm{Sa}, \mathrm{Vi}$ & Ds \\
\hline $\begin{array}{l}\text { Buzzell, Foss \& Middleton } \\
\text { (2006) }\end{array}$ & $\begin{array}{l}\text { universitários } \\
\text { (SF/SM) }\end{array}$ & 134 & desvio a ser controlado & - & Sx \\
\hline Chi et al. (2012) & $\begin{array}{l}\text { universitários } \\
\text { (SF/SM) }\end{array}$ & 1403 & $\begin{array}{l}\text { sem definição, com } \\
\text { exemplos }\end{array}$ & - & Ds \\
\hline $\begin{array}{l}\text { Crimmins \& Seigfried- } \\
\text { Spellar (2014) }\end{array}$ & $\begin{array}{l}\text { universitários } \\
\text { (SF/SM) }\end{array}$ & 88 & sem definição & Sa & Sx \\
\hline D’Abreu (2013) & $\begin{array}{l}\text { universitários } \\
\text { (SM) } \\
\text { casais }\end{array}$ & 329 & $\begin{array}{l}\text { material sexualmente } \\
\text { explícito para excitação }\end{array}$ & Re-, Vi & - \\
\hline $\begin{array}{l}\text { Daneback, Træen \& } \\
\text { Månsson (2009) }\end{array}$ & $\begin{array}{l}\text { heterossexuais } \\
\text { (casados ou } \\
\text { morando juntos) }\end{array}$ & 398 & não há consenso & Sa, Re- & Sx \\
\hline DeKeseredy (2015) & RL & $\mathrm{N} / \mathrm{A}$ & $\begin{array}{l}\text { material sexualmente } \\
\text { explícito, com } \\
\text { submissão feminina } \\
\text { material sexualmente }\end{array}$ & $\mathrm{Vi}, \mathrm{Sc}$ & - \\
\hline El Far (2007) & RL & $\mathrm{N} / \mathrm{A}$ & $\begin{array}{l}\text { explícito, fere bons } \\
\text { costumes }\end{array}$ & $\mathrm{Sa}, \mathrm{Sc}$ & - \\
\hline Elder et al. (2015) & homossexuais & 20 & sem definição & $\mathrm{Sa}, \mathrm{Sc}$ & Sx, Ds, Re+ \\
\hline Fahs \& Gonzalez (2014) & mulheres & 20 & $\begin{array}{l}\text { sem definição, com } \\
\text { exemplos }\end{array}$ & Sc & Sx \\
\hline $\begin{array}{l}\text { Gorman, Monk-Turner \& } \\
\text { Fish (2010) }\end{array}$ & vídeos* & $\mathrm{N} / \mathrm{A}$ & $\begin{array}{l}\text { material sexualmente } \\
\text { explícito, com } \\
\text { submissão feminina }\end{array}$ & Vi & - \\
\hline
\end{tabular}

A Gerais: Revista Interinstitucional de Psicologia, 13(1), 2020, el30103 


\begin{tabular}{|c|c|c|c|c|c|}
\hline Grov et al. (2011) & $\begin{array}{l}\text { adultos }(\mathrm{SF} / \mathrm{SM}) \\
\text { em um } \\
\text { relacionamento }\end{array}$ & 8376 & sem definição & Sa, Re- & Sx, Ds, Re+ \\
\hline $\begin{array}{l}\text { Gwinn, Lambert, } \\
\text { Fincham \& Maner (2013) }\end{array}$ & $\begin{array}{l}\text { universitários } \\
(\mathrm{SF} / \mathrm{SM}) \text { em um } \\
\text { relacionamento }\end{array}$ & $\begin{array}{l}74 / \\
291\end{array}$ & $\begin{array}{l}\text { sem definição, com } \\
\text { exemplos }\end{array}$ & Re- & - \\
\hline $\begin{array}{l}\text { Hald, Malamuth \& Yuen } \\
\text { (2010) }\end{array}$ & MA & $\mathrm{N} / \mathrm{A}$ & $\begin{array}{l}\text { material sexualmente } \\
\text { explícito para excitação }\end{array}$ & $\mathrm{Vi}$ & - \\
\hline Hald \& Malamuth (2015) & adultos (SF/SM) & 200 & sem definição & $\mathrm{Vi}$ & - \\
\hline Hearn (2006) & RL & $\mathrm{N} / \mathrm{A}$ & sem definição & Re-, Vi & - \\
\hline Hilton (2013) & RL & $\mathrm{N} / \mathrm{A}$ & sem definição & $\mathrm{Sa}, \mathrm{Vi}$ & - \\
\hline $\begin{array}{l}\text { Laier, Pekal \& Brand } \\
\text { (2014) }\end{array}$ & mulheres & 102 & material hard-core & - & - \\
\hline Levine (2010) & $\begin{array}{l}\text { homens } \\
\text { casal }\end{array}$ & 30 & sem definição & Re- & - \\
\hline Limacher \& Wright (2006) & $\begin{array}{c}\text { heterossexual } \\
\text { em terapia } \\
\text { adultos (SF/SM) }\end{array}$ & 1 & sem definição & Re- & - \\
\hline Maddox et al. (2011) & $\begin{array}{l}\text { em um } \\
\text { relacionamento, } \\
\text { não casados }\end{array}$ & 1291 & $\begin{array}{l}\text { material sexualmente } \\
\text { explícito }\end{array}$ & Re- & Sx, Re+ \\
\hline Montero (2008) & RL & $\mathrm{N} / \mathrm{A}$ & $\begin{array}{l}\text { material sexualmente } \\
\text { explícito }\end{array}$ & Sc & - \\
\hline Mowlabocus et al. (2013) & homossexuais & $\begin{array}{c}125 / \\
50\end{array}$ & $\begin{array}{l}\text { material homossexual } \\
\text { sexualmente explícito }\end{array}$ & Sc & Sx, Ds \\
\hline Muusses et al. (2015) & $\begin{array}{c}\text { casais } \\
\text { heterossexuais } \\
\text { casados }\end{array}$ & $\begin{array}{c}190 / \\
157 / \\
140\end{array}$ & $\begin{array}{l}\text { material sexualmente } \\
\text { explícito }\end{array}$ & Re- & $\operatorname{Re}+$ \\
\hline Olmstead et al. (2013) & $\begin{array}{l}\text { universitários } \\
\text { (SF/SM) }\end{array}$ & 404 & $\begin{array}{l}\text { material sexualmente } \\
\text { explícito }\end{array}$ & Sa, Re- & Sx, Re+ \\
\hline Poll (2012) & RL & $\mathrm{N} / \mathrm{A}$ & $\begin{array}{l}\text { material sexualmente } \\
\text { explícito }\end{array}$ & $\mathrm{Vi}$ & - \\
\hline Popović (2011) & mulheres & 66 & $\begin{array}{l}\text { material sexualmente } \\
\text { explícito para excitação }\end{array}$ & Sa, Re- & $\mathrm{Sx}, \mathrm{Re}+$ \\
\hline Pyle \& Bridges (2012) & $\begin{array}{l}\text { universitárias } \\
\text { (SF) }\end{array}$ & 168 & sem definição & Sa, Re- & - \\
\hline Şenormanci et al. (2014) & homens & 2 & sem definição & $\mathrm{Sa}$ & - \\
\hline Silvera et al. (2015) & homossexuais & 1995 & $\begin{array}{l}\text { material sexualmente } \\
\text { explícito para excitação }\end{array}$ & $\mathrm{Sa}$ & Ds \\
\hline Staley \& Prause (2013) & $\begin{array}{l}\text { casais } \\
\text { heterossexuais }\end{array}$ & 44 & $\begin{array}{l}\text { material sexualmente } \\
\text { explícito }\end{array}$ & Sa & Sx, Ds \\
\hline
\end{tabular}

A Gerais: Revista Interinstitucional de Psicologia, 13(1), 2020, el30103 


\begin{tabular}{|c|c|c|c|c|c|}
\hline $\begin{array}{l}\text { Stewart } \quad \& \quad \text { Szymanski } \\
(2012)\end{array}$ & $\begin{array}{l}\text { universitárias } \\
\text { (SF) }\end{array}$ & 308 & $\begin{array}{l}\text { material sexualmente } \\
\text { explícito para excitação }\end{array}$ & Sa, Re- & - \\
\hline $\begin{array}{l}\text { Sun, Miezan, Lee \& Shim } \\
\text { (2015) }\end{array}$ & homens & 685 & $\begin{array}{l}\text { material sexualmente } \\
\text { explícito para excitação }\end{array}$ & Sa, Re- & Sx, Re+ \\
\hline Szymanski et al. (2015) & $\begin{array}{l}\text { universitárias } \\
\text { (SF) }\end{array}$ & 359 & $\begin{array}{l}\text { material sexualmente } \\
\text { explícito }\end{array}$ & Sa, Re- & - \\
\hline Tylka (2015) & $\begin{array}{l}\text { universitários } \\
\text { (SM) }\end{array}$ & 171 & $\begin{array}{l}\text { sem definiçãa, com } \\
\text { exemplos }\end{array}$ & $\mathrm{Sa}, \mathrm{Re}-\mathrm{S} \mathrm{Sc}$ & - \\
\hline Tylka \& Van Diest (2015) & $\begin{array}{l}\text { universitárias } \\
\text { (SF) }\end{array}$ & 359 & $\begin{array}{l}\text { sem definição, com } \\
\text { exemplos }\end{array}$ & $\mathrm{Sa}, \mathrm{Vi}, \mathrm{Sc}$ & - \\
\hline $\begin{array}{l}\text { Weinberg, Williams, } \\
\text { Kleiner \& Irizarry (2010) }\end{array}$ & $\begin{array}{l}\text { universitários } \\
(\mathrm{SF} / \mathrm{SM})\end{array}$ & $\begin{array}{c}172 / \\
73\end{array}$ & $\begin{array}{l}\text { material sexualmente } \\
\text { explícito }\end{array}$ & - & Sx, Ds \\
\hline Weinstein et al. (2015) & adultos (SF/SM) & 267 & sem definição & $\mathrm{Sa}, \mathrm{Re}-, \mathrm{Sc}$ & - \\
\hline Wentland \& Muise (2010) & fotógrafos & 11 & $\begin{array}{l}\text { material sexualmente } \\
\text { explícito para excitação }\end{array}$ & - & Sx, Ds \\
\hline Wu et al. (2014) & $\begin{array}{l}\text { homens } \\
\text { migrantes }\end{array}$ & 4069 & $\begin{array}{l}\text { sem definição, com } \\
\text { exemplos }\end{array}$ & Sa & - \\
\hline Wulandari (2015) & $\mathrm{RL}$ & $\mathrm{N} / \mathrm{A}$ & sem definição & - & - \\
\hline Yucel \& Gassanov (2010) & $\begin{array}{c}\text { casais } \\
\text { heterossexuais } \\
\text { casados }\end{array}$ & 433 & $\begin{array}{l}\text { sem definição, com } \\
\text { exemplos }\end{array}$ & Re- & - \\
\hline
\end{tabular}

Nota: * pesquisa realizada com vídeos disponibilizados em sites com conteúdo adulto. RL = revisão de literatura;

$\mathrm{MA}=$ meta-análise; $\mathrm{N} / \mathrm{A}=$ não se aplica (estudos que não tiveram participantes identificáveis); SF = sexo feminino;

$\mathrm{SM}=$ sexo masculino. Amostras com números separados por / indicam pesquisas com múltiplas etapas e o respectivo número de participantes por etapa. Categorias dos aspectos negativos: (Sa) prejuízos à saúde, (Re-) prejuízos ao relacionamento, (Vi) violência contra as mulheres e ( $\mathrm{Sc}$ ) aspectos socioculturais. Categorias dos aspectos positivos: (Sx) sexualidade, (Ds) desenvolvimento pessoal e $(\mathrm{Re}+)$ benefícios ao relacionamento.

Fonte: Elaborado pelos autores. 
Observa-se um maior número de pesquisas com pessoas em relacionamento $(\mathrm{N}=$ 9; 24\%), nas quais o pré-requisito para os participantes era estar em um relacionamento amoroso (definidos nos artigos de diferentes formas, tais como casamento, união civil, relacionamento romântico, íntimo ou relação de compromisso). Esse índice pode ter sido encontrado em virtude dos termos "relacionamento" e "casal" fazerem parte da estratégia de busca dos artigos. Poucas pesquisas focaram na população homossexual, e apenas masculina $(\mathrm{N}=3 ; 8 \%)$. Uma pesquisa trabalhou exclusivamente com fotógrafos especializados em fotografias eróticas, buscando compreender a motivação dos clientes - homens, mulheres e casais - e o processo de produção dessas fotos (Wentland \& Muise, 2010).

Quanto ao número de participantes, nota-se que pesquisas com mais de 100 respondentes representam $70 \%(\mathrm{~N}=26)$ dos artigos, indicando que as pesquisas foram, em sua maioria, com grandes amostras. Tal dado coaduna com o tipo de estudo realizado: 23 utilizaram coleta exclusivamente quantitativas $(62 \%)$ e seis utilizaram coleta mistas $(16 \%)$.

Buscando identificar e descrever como a pornografia tem sido definida no meio científico, foi levantada em cada um dos artigos selecionados a definição de pornografia com a qual os autores trabalharam: $47 \%$ definem como material sexualmente explícito (nove apenas como isso; sete acrescem o objetivo de excitar o consumidor; cinco, ainda, usam outros diferenciais , como "ferindo os bons-costumes", "sem intimidade" ou "com submissão feminina", por exemplo), 44\% dos artigos não apresentam uma definição de pornografia (12 não dão definição alguma; oito não definem, mas dão exemplos: vídeo, revista, foto, site de Internet, etc.), 4\% descreve como material exclusivamente hard-core (com imagens de violência, como asfixia, engasgos, etc.), $2 \%$ como material desviante e $2 \%$ explicita que não há consenso na definição.

Como pode ser observado no Quadro 1, alguns autores partem do pressuposto de que há um consenso no que se avalia como pornográfico, não explicitando nenhuma definição ou se limitando a exemplos. Outros, ainda, restringem a avaliação do que é pornográfico àqueles conteúdos extremos, violentos. $\mathrm{Na}$ amostra estudada, material sexualmente explícito parece ser a definição mais aceita, independentemente das gradações e diferentes tipos/categorias de pornografia.

Mostra-se interessante o surgimento das palavras erotismo e fotografia erótica, usadas nos artigos como algo distinto da pornografia por sua explicitude (Montero, 2008) e como sinônimo, intentando despertar a excitação (Wentland \& Muise, 2010), respectivamente. Tal possibilidade demonstra a maleabilidade dos conceitos pornografia e erotismo, e a diversidade de termos usados para identificar a pornografia.

Para auxiliar na compreensão dos efeitos ao relacionamento amoroso do uso de pornografia, e levando em consideração a dualidade já percebida nessa interface, foram identificados em cada artigo aspectos negativos e positivos desse consumo apontados pelos autores. Esses aspectos foram agrupados em categorias que podem ser identificadas no Quadro 1, discutidas a seguir. O levantamento dos aspectos positivos e negativos e sua categorização foram feitos por dois juízes independentes, com índice de concordância de 
$70 \%$. Aspectos discordantes foram analisados por um terceiro juiz, que promoveu o consenso.

Aspectos negativos percebidos do consumo de pornografia ao relacionamento foram encontrados em 39 artigos (87\%) e foram agrupados nas seguintes categorias:

1. Prejuízos à saúde (32\%): expressões que indicam prejuízos percebidos à saúde física e mental, tais como uso problemático, autopercepsão negativa, comportamento sexual de risco, etc. Destacase aqui a preocupação com o desenvolvimento de um vício e suas consequências, bem como aspectos relacionados ao sofrimento psíquico e seus efeitos.

2. Prejuízos ao relacionamento $(31 \%)$ : termos vinculados ao relacionamento, tais como redução da satisfação, segredo, infidelidade, redução da intimidade, etc. Denota as ameaças à manutenção da relação e as perdas sofridas por ela em virtude do consumo de pornografia por um ou ambos os companheiros.

3. Violência contra as mulheres $(21 \%)$ : enfocam a preocupação de que a pornografia possa ser um catalisador de relações desiguais, como violência contra a mulher, objetificação, submissão feminina, etc. Trata da forma como a mulher é vista majoritariamente na pornografia: como um objeto sexual para satisfação masculina.

4. Aspectos socioculturais (16\%): referências a aspectos sociais e culturais que influenciam nossos comportamentos sexuais, especialmente por meio de uma comparação com o outro - a idealização, de como o corpo deve ser, de como o desempenho deve acontecer, etc.

Algumas evidências sugerem que mulheres e, mais frequentemente, homens podem ser "atraídos" para a pornografia quando se sentem solitários e frustrados sexualmente
(Popovic, 2011; Muusses et al., 2015). Um dos estudos buscou compreender a direcionalidade da relação entre o uso de material sexualmente explícito na internet e a qualidade do relacionamento entre casais e conclui indicando que enquanto esposas usam pornografia para apimentar sua vida sexual, maridos usam como forma de demostrar que não estão satisfeitos na relação (Muusses et al., 2015). Isso nos leva a ponderar que existem diferenças de gênero significativas no consumo de pornografia e, pensando em termos de causalidade, que talvez alguns dos prejuízos percebidos sejam reflexos da condição da relação, especialmente quando o casal já tem algum problema prévio relativo à confiança, ao sexo ou, ainda, quando os parceiros têm uma percepção negativa da pornografia em si (Popovic, 2011; Stewart \& Szymanski, 2012).

Pesquisas indicam que o uso excessivo de pornografia pode ter impactos negativos no relacionamento (Stewart \& Szymanski, 2012; Hilton, 2013; Olmstead et al., 2013; Şenormanci et al., 2014); algumas destas utilizam como população sujeitos com consumo patológico como o uso dessas pessoas está acima da média da população, supõe-se que suas atitudes relativas a isso também estarão fora da média. Faz-se mister, então, a atenção na recepção dos resultados desses estudos, de modo a não generalizar para a população geral os efeitos encontrados entre pessoas que fazem uso excessivo de pornografia. Pyle e Bridges (2012) buscaram investigar se os efeitos negativos da pornografia são diferentes dos produzidos por outros comportamentos compulsivos, como uso de maconha. Concluíram que a maior frequência do uso, maior segredo e menor disponibilidade do parceiro para interação foram os fatores que, de 
forma similar nos dois tipos de comportamento, impactaram os relacionamentos. Parece que, independentemente da experiência em análise, o uso excessivo e, notadamente, o segredo, tende a criar problemas na relação (Limacher \& Wright, 2006; Levine, 2010; Popovic, 2011).

Uma das preocupações com o uso de pornografia é que esta, ao reproduzir a expressão da cultura patriarcal do mundo atual, possa influenciar a compreensão dos papéis de gênero e comportamentos sexuais esperados em um relacionamento, partindo da premissa de subordinação das mulheres, normatizando-as, contribuindo, assim, com situações de objetificação e de violência (Bonomi et al., 2014; Braithwaite et al., 2015; DeKeseredy, 2015; Hald \& Malamuth, 2015).

Essa "reprodução" das condutas socialmente aceitas não se limita ao relacionamento romântico - são voltadas para ele, mas também tem efeitos na internalização daquilo que é retratado nos meios midiáticos. Alguns dos artigos selecionados apontam a idealização do setting pornográfico, resultando em pressão pelos próprios sujeitos em alcançarem um corpo ideal, um desempenho ideal, uma relação sexual ideal com um parceiro ideal (Bonomi et al., 2014; DeKeseredy, 2015; Elder et al., 2015; Tylka \& Van Diest, 2015) - todos inalcançáveis, gerando uma percepção de si negativa e ansiedade e insegurança no estabelecimento de relações mais próximas (El Far, 2007; Grov et al., 2011; Staley \& Prause, 2013; Tylka, 2015). Uma das pesquisas aponta, ainda, que homens em relacionamento tendem a preferir a pornografia ao sexo real quando comparados, o pornô parece mais emocionante, com sua fonte infinita de mulheres e atos sexuais diversificados (Sun et al., 2015). É importante observar que esses modelos idealizados não são exclusivos da pornografia, mas permeiam toda produção midiática.

Aspectos positivos percebidos do consumo de pornografia ao relacionamento foram encontrados em 19 artigos (42\%) e foram agrupados nas seguintes categorias:

1. Sexualidade (44\%): expressões que indicam benefícios à vida sexual e ao desenvolvimento da sexualidade, como diversificação sexual, apimentar a relação, maior satisfação sexual, etc. São efeitos diretos na vida sexual dos indivíduos, além de incorporado pelos casais em suas relações sexuais.

2. Desenvolvimento pessoal (31\%): referências à diversificação dos recursos pessoais por meio da utilização de pornografia, como aprendizado, normalização, autoestima, confiança, etc. Remete à ampliação dos horizontes sexuais com o pornô como fonte de aprendizado, de normalização e validação de desejos e fantasias e de avaliação positiva de si e do próprio comportamento sexual.

3. Benefícios ao relacionamento $(25 \%)$ : termos vinculados ao relacionamento, tais como: melhor comunicação, maior dedicação, maior intimidade, etc. Aponta as possíveis contribuições do consumo de pornografia ao relacionamento, como uma forma de maior aproximação dos pares.

Na pesquisa de Staley e Prause (2013), foram investigados os efeitos na excitação de adultos (homens e mulheres) após a exibição de um de dois tipos de filme: erótico ou emocionante (não erótico). Ambos resultaram em aumento equivalente na excitação, sendo o erótico classificado como um pouco mais excitante e provocando no participante maior 
desejo de estar com o parceiro. Supõe-se, dessa maneira, que o efeito excitatório não se restringe aos filmes de sexo explícito.

Para muitas pessoas, a pornografia parece ser a principal fonte de informação sexual "pornô é como uma escola televisionada para o sexo" (fala de um participante da pesquisa de Elder et al., 2015). Auxilia na diversificação das práticas sexuais (Chi et al., 2012; Mowlabocus et al., 2013; Olmstead et al., 2013; Elder et al., 2015), na confrontação e normalização dos desejos (Weinberg et al., 2010; Elder et al., 2015). Também é ferramenta para que os casais falem sobre suas fantasias (Fahs \& Gonzalez, 2014) e para a comunicação na relação, aproximando os parceiros (Benjamin \& Tlusten, 2010; Grov et al., 2011; Popovic, 2011; Elder et al., 2015).

Quer se tome a pornografia numa perspectiva mais negativa, de implicações morais, exploração, danos e riscos, ou positiva, como ampliação das possibilidades sexuais e desenvolvimento pessoal, o que se percebe é um fenômeno complexo. Trata da sexualidade humana, algo multifacetado e culturalmente construído - e sobre o qual é preciso lançar luz para que possamos melhor compreendê-lo e, assim, utilizá-lo na construção de uma sexualidade humana mais potente e saudável.

\section{Considerações Finais}

A utilização de revisão sistemática forneceu um panorama sobre o tema, destacando a diversidade de aspectos tratados, e buscou construir informações que, disseminadas, possam contribuir com a ampliação do conhecimento científico sobre o assunto e com as estratégias de intervenção utilizadas pelos profissionais de saúde, especialmente psicólogos. O discurso sobre sexo e sua exposição na mídia, de formas veladas e explícitas, é constante, permeando desde programas de auditório a propagandas de cerveja, e influenciando a forma como as pessoas se relacionam.

Os possíveis impactos do consumo de pornografia nos relacionamentos são ainda bastante imprecisos e ambíguos. Relatórios clínicos, opinião pública e pesquisas com dados variam muito no que se referem aos presumidos efeitos negativos e positivos desse tipo de material, e ora são apresentados como risco de dissolução da relação (Maddox et al., 2011; Popovic, 2011; Olmstead et al., 2013; Weinstein et al., 2015), ora como fortalecedor do relacionamento (Benjamin \& Tlusten, 2010; Grov et al., 2011; Popovic, 2011; Elder et al., 2015). Por diversas vezes, inclusive, os artigos apresentam ambas as possibilidades.

Os resultados apontam uma discrepância entre a sexualidade "representada" e a "real"; sugerem, ainda, que a pornografia pode ser uma fonte de informação básica sobre sexo e pode ter alguma influência sobre as práticas sexuais dos inquiridos. É preciso ampliar os canais para que se possa falar sobre a sexualidade humana, contribuindo para vivências menos limitadoras e mais saudáveis, encorajando as pessoas a discutirem sobre os possíveis benefícios e efeitos nocivos do uso desse tipo de material e o que leva ao consumo (Chi et al., 2012).

A proposta de investigar as definições de pornografia utilizadas por pesquisadores e os efeitos percebidos do consumo desse material nos relacionamentos amorosos foi alcançada. Entretanto, duas possíveis limitações podem ser apontadas: a escolha das bases para busca 
depende da disponibilidade e da possibilidade de se refinar a pesquisa, para atender aos critérios de seleção, e tornar a investigação viável; e a multiplicidade de interpretações dos termos de busca gera retorno de muitos documentos que são excluídos da base de dados final.

Duas questões principais instigam a continuidade desta investigação, para além dessa pesquisa: 1. Qual o limiar entre a pornografia dita "prejudicial" e "benéfica" e como as características pessoais (sentimentos, valores, crenças, expectativas) contribuem nas atitudes relativas a esse material; e 2. Como esse fenômeno é manifestado no contexto brasileiro, com suas características sociais, culturais, históricas e econômicas próprias.

\section{Referências}

Andrade, A. L., Garcia, A., \& Cano, D. S. (2009). Preditores da satisfação global em relacionamentos românticos. Psicologia: Teoria e Prática, 11(3), 143-156. Recuperado de

http://pepsic.bvsalud.org/pdf/ptp/v11n 3/v11n3a12.pdf.

Associação Brasileira das Empresas do Mercado Erótico e Sensual (2014). Dados estatísticos. Recuperado de www.abeme.com.br/publicacoes_old/dad os-estatisticos/.

Benjamin, O., \& Tlusten, D. (2010). Intimacy and/or degradation: heterosexual images of togetherness and women's embracement of pornography. Sexualities, 13(5), 599-623. Doi: 10.1177/1363460710376492.

Bonomi, A. E., Nemeth, J. M., Altenburger, L. E., Anderson, M. L., Snyder, A., \& Dotto, I. (2014). Fiction or not? Fifty shades is associated with health risks in adolescent and young adult females. Journal of Women's Health (2002), 23(9), 720-728. Doi: 10.1089/jwh.2014.4782.
Bouffard, L. A. (2010). Exploring the utility of entitlement in understanding sexual aggression. Journal of Criminal Justice, 38(5), 870-879

Doi: 10.1016/j.jcrimjus.2010.06.002.

Braithwaite, S., Aaron, S., Dowdle, K., Spjut, K., \& Fincham, F. (2015). Does pornography consumption increase participation in friends with benefits relationships? Sexuality \& Culture, 19(3), 513-532. Doi: 10.1007/s12119-015-9275-4.

Buzzell, T., Foss, D., \& Middleton, Z. (2006). Explaining use of online pornography: A test of self-control theory and opportunities for deviance. Journal of Criminal Justice and Popular Culture, 13(2), 96116. Retrieved from www.albany.edu/scj/jcjpc/vol13is2/Buzz ell.pdf.

Chi, X., Yu, L., \& Winter, S. (2012). Prevalence and correlates of sexual behaviors among university students: a study in Hefei, China. BMC Public Health, 12(972). Doi: 10.1186/1471-2458-12-972.

Costa, A. B., \& Zoltowski, A. P. C. (2014). Como escrever um artigo de revisão sistemática. In S. H. Koller, M. C. P. P. Couto \& Hohendorff, J. V. Manual de producão científica (pp. 55-70). Porto Alegre: Penso.

Crimmins, D. M., \& Seigfried-Spellar, K. C. (2014). Peer attachment, sexual experiences, and risky online behaviors as predictors of sexting behaviors among undergraduate students. Computers in Human Behavior, 32, 268-275. Doi: 10.1016/j.chb.2013.12.012.

D’Abreu, L. C. F. (2013). Pornografia, desigualdade de gênero e agressão sexual contra mulheres. Psicologia \& Sociedade, 25(3), 592-601. Recuperado de http://www.ufrgs.br/seerpsicsoc/ojs2/in dex.php/seerpsicsoc/article/view/3658/ 2274.

Daneback, K., Træen, B., \& Månsson, S. (2009). Use of pornography in a random sample of norwegian heterosexual couples. Archives of Sexual Bebavior, 38(5), 746-753. Doi: 10.1007/s10508-008-9314-4. 
DeKeseredy, W. (2015). Critical Criminological Understandings of Adult Pornography and Woman Abuse: New Progressive Directions in Research and Theory. International Journal For Crime, Justice and Social Democracy, 4(4), 4-21. Doi: 10.5204/ijcjsd.v4i4.184.

El Far, A. (2007). Crítica social e ideias médicas nos excessos do desejo: uma análise dos "romances para homens" de finais do século XIX e início do XX. Cadernos Pagu, 28, 285-312. Recuperado de http://www.scielo.br/pdf/cpa/n28/13.pd f.

Elder, W. B., Morrow, S. L., \& Brooks, G. R. (2015). Sexual self-schemas of gay men: a qualitative investigation. The Counseling Psychologist, $\quad 43(7), \quad 942-969 . \quad$ Doi: 10.1177/0011000015606222.

Fahs, B., \& Gonzalez, J. (2014). The front lines of the "back door": navigating (dis)engagement, coercion, and pleasure in women's anal sex experiences. Feminism and Psychology, 24(4), 500-520. Doi: $10.1177 / 0959353514539648$.

Gorman, S., Monk-Turner, E., \& Fish, J. N. (2010). Free adult internet web sites: how prevalent are degrading acts?. Gender Issues, 27(3-4), 131-145. Doi: 10.1007/s12147010-9095-7.

Grov, C., Gillespie, B. J., Royce, T., \& Lever, J. (2011). Perceived consequences of casual online sexual activities on heterosexual relationships: a U.S. online survey. Archives of Sexual Behavior, 40(2), 429-439. Doi: 10.1007/s10508-010-9598-z.

Guerra, V. M., Andrade, F. C. B., \& Dias, M. R. (2004). Atitudes de estudantes universitários frente ao consumo de materiais pornográficos. Estudos de Psicologia, 9(2), 269-277. Doi: 10.1590/S1413-294X2004000200008.

Gwinn, A. M., Lambert, N. M., Fincham, F. D., \& Maner, J. K. (2013). Pornography, relationship alternatives, and intimate extradyadic behavior. Social Psychological and Personality Science, 4(6), 699-704. Doi: 10.1177/1948550613480821.
Hald, G. M., Malamuth, N. M., \& Yuen, C. (2010). Pornography and attitudes supporting violence against women: revisiting the relationship in nonexperimental studies. Aggressive Behavior, 36(1), 14-20. Doi: 10.1002/ab.20328.

Hald, G. M., \& Malamuth, N. M. (2015). Experimental effects of exposure to pornography: the moderating effect of personality and mediating effect of sexual arousal. Archives of Sexual Behavior, 44(1), 99-109. Doi: 10.1007/s10508-014-0291-5.

Hearn, J. (2006). The implications of information and communication technologies for sexualities and sexualised violences: contradictions of sexual citizenships. Political Geography, 25(8), 944-963. Doi: 10.1016/j.polgeo.2006.08.007.

Hilton, D. (2013). Pornography addiction - A supranormal stimulus considered in the context of neuroplasticity. Socioaffective Neuroscience \& Psychology, 3. Doi: 10.3402/snp.v3i0.20767.

Laier, C., Pekal, J., \& Brand, M. (2014). Cybersex addiction in heterosexual female users of internet pornography can be explained by gratification hypothesis. Cyberpsychology, Behavior, and Social Networking, 17(8), 505511. Doi: 10.1089/cyber.2013.0396.

Levine, S. B. (2010). What is sexual addiction?. Journal of Sex and Marital Therapy, 36(3), 261-275.

Doi: $10.1080 / 00926231003719681$.

Limacher, L. H., \& Wright, L. M. (2006). Exploring the therapeutic family intervention of commendations: insights from research. Journal of Family Nursing, 12(3), 307-331. Doi: 10.1177/1074840706291696.

Lins, R. N. (2012). O livro do amor, volume 1: da Prébistória à Renascença. Rio de Janeiro: Best Seller.

Maddox, A. M., Rhoades, G. K., \& Markman, H. J. (2011). Viewing sexually-explicit materials alone or together: associations with relationship quality. Archives of Sexual Behavior, 40(2), 441-448. Doi: 10.1007/s10508-009-9585-4. 
Michaelis (2015). Dicionário Brasileiro da Lingua Portuguesa. São Paulo: Editora Melhoramentos. Recuperado de https://michaelis.uol.com.br/modernoportugues/.

Montero, S. G. (2008). Del erotismo a la seducción: en torno a Kant y Kierkegaard. Revista Observaciones Filosóficas, 7. Recuperado de http://www.observacionesfilosoficas.net/ delerotismoalaseduccion.html.

Mowlabocus, S., Harbottle, J., \& Witzel, C. (2013). Porn laid bare: gay men, pornography and bareback sex. Sexualities, 16(5-6), 523-547. Doi: $10.1177 / 1363460713487370$.

Muusses, L. D., Kerkhof, P., \& Finkenauer, C. (2015). Internet pornography and relationship quality: a longitudinal study of within and between partner effects of adjustment, sexual satisfaction and sexually explicit internet material among newlyweds. Computers in Human Behavior, 45, 7784. Doi: 10.1016/j.chb.2014.11.077.

Olmstead, S. B., Negash, S., Pasley, K., \& Fincham, F. D. (2013). Emerging adults' expectations for pornography use in the context of future committed romantic relationships: a qualitative study. Archives of Sexual Behavior, 42(4), 625-635. Doi: 10.1007/s10508-012-9986-7.

Poll, L. V. D. (2012). But is it speech? Making critical sense of the dominant constitutional discourse on pornography, morality and harm under the pervasive influence of United States first amendment jurisprudence. Potchefstroom Electronic Law Journal, 15(2), 416-452. Doi: 10.4314/pelj.v15i2.15.

Popović, M. (2011). Pornography use and closeness with others in women. Srpski Arbiv za Celokupno Lekarstvo, 139(5-6), 353359. Doi: 10.2298/SARH1106353P.

Pornhub Team (2018, December, 11). 2018 Year in Review. Pornbub Insights. Retrieved from www.pornhub.com/insights/2018-yearin-review.

Pyle, T. M., \& Bridges, A. J. (2012). Perceptions of relationship satisfaction and addictive behavior: comparing pornography and marijuana use. Journal of Behavioral Addictions, 1(4), 171-179. Doi: 10.1556/JBA.1.2012.007.

Şenormanci, Ö., Konkan, R., Güçlü, O., \& Şenormanci, G. (2014). Two cases of excessive internet use with comorbid family relationship problems. Noropsikijatri Arsivi, 51(3), 280-282. Doi: 10.4274/npa.y6939.

Silvera, R. J., Grov, C., Stein, D. J., Hagerty, R., \& Marmor, M. (2015). Level of "outness" and pornography use among men who have sex with men: results from an online survey. Psychology \& Sexuality, 6(1), 44-58. Doi: 10.1080/19419899.2014.984907.

SimilarWeb (2018, November, 01). Top Websites Ranking. Resources. Retrieved from www.similarweb.com/top-websites.

Staley, C., \& Prause, N. (2013). Erotica viewing effects on intimate relationships and self/partner evaluations. Archives of Sexual Behavior, 42(4), 615-624. Doi: 10.1007/s10508-012-0034-4.

Stewart, D. N., \& Szymanski, D. M. (2012). Young adult women's reports of their male romantic partner's pornography use as a correlate of their self-esteem, relationship quality, and sexual satisfaction. Sex Roles, 67(5-6), 257-271. Doi: 10.1007/s11199012-0164-0.

Sun, C., Miezan, E., Lee, N., \& Shim, J. W. (2015). Korean men's pornography use, their interest in extreme pornography, and dyadic sexual relationships. International Journal of Sexual Health, 27(1), 16-35. Doi: 10.1080/19317611.2014.927048.

Szymanski, D. M., Feltman, C. E., \& Dunn, T. L. (2015). Male partner's perceived pornography use and women's relational and psychological health: the roles of trust, attitudes, and investment. Sex Roles, 73(56), 187-199. Doi: 10.1007/s11199-0150518-5.

Tylka, T. L. (2015). No harm in looking, right? Men's pornography consumption, body image, and well-being. Psychology of Men \& Masculinity, 16(1), 97-107. Doi: 10.1037/a0035774. 
Tylka, T. L., \& Van Diest, A. M. K. (2015). You looking at her "hot" body may not be "cool" for me: integrating male partners' pornography use into objectification theory for women. Psychology of Women Quarterly, 39(1), 67-84. Doi: $10.1177 / 0361684314521784$.

Weinberg, M. S., Williams, C. J., Kleiner, S., \& Irizarry, Y. (2010). Pornography, normalization, and empowerment. Archives of Sexual Behavior, 39(6), 1389-1401. Doi: 10.1007/s10508-009-9592-5.

Weinstein, A. M., Zolek, R., Babkin, A., Cohen, K., \& Lejoyeux, M. (2015). Factors predicting cybersex use and difficulties in forming intimate relationships among male and female users of cybersex. Frontiers in Psychiatry, 6(54), 1-8. Doi: 10.3389/fpsyt.2015.00054.

Wentland, J. J., \& Muise, A. (2010). Stepping out from behind the lens: a qualitative analysis of erotic photographers. Sexuality and Culture, 14(2), 97-125. Doi: 10.1007/s12119-010-9068-8.

Wu, J. Q., Wang, K. W., Zhao, R., Li, Y. Y., Zhou, Y., Li, Y. R., Ji, H. L., \& Ji, M. (2014). Male rural-to-urban migrants and risky sexual behavior: a cross-sectional study in Shanghai, China. International Journal of Environmental Research and Public Health, 11(3), 2846-2864. Doi: 10.3390/ijerph110302846.

Wulandari, E. (2015). Pluralism in setting and signification of pornography. Jurnal Dinamika Hukum, 15(1), 111-119. Retrieved from http://dinamikahukum.fh.unsoed.ac.id/in dex.php/JDH/article/viewFile/401/367.

Yucel, D., \& Gassanov, M. A. (2010). Exploring actor and partner correlates of sexual satisfaction among married couples. Social Science Research, 39(5), 725-738. Doi: 10.1016/j.ssresearch.2009.09.002.

Recebido em: 13/4/2017

Aprovado em: 23/12/2018 